PACS 72.20.Jv, 88.40.hj, jj

\title{
The study of solar cells with back side contacts at low illumination
}

\author{
A.P. Gorban, V.P. Kostylyov, V.G. Litovchenko, A.V. Sachenko, A.A. Serba, \\ I.O. Sokolovskyi, V.V. Chernenko \\ V. Lashkaryov Institute of Semiconductor Physics, NAS of Ukraine \\ 45, prospect Nauky, 03028 Kyiv, Ukraine; e-mail: sach@isp.kiev.ua
}

\begin{abstract}
Theoretical analysis and experimental research of $\mathrm{Si}$ solar cells (SC) with interdigitated back side contacts (BSC) photovoltaic parameters and photoconversion efficiency at low light level have been done in presence of floating $p^{+}-n$ junctions and isotype $n^{+}-n$ junctions on frontal (illuminated) surface. It has been found that in case of floating junction the magnitudes of short-circuit current, open-circuit voltage and efficiency, as well as of internal quantum efficiency of photocurrent can decrease significantly due to recombination in the space charge region (SCR) rather than to surface recombination. In case of isotype junction, this decrease is absent. These results allow to conclude that the floating $p^{+}-n$ junctions at the front surface of the silicon BSC SC would be appropriate for use only in case of an illumination intensity $\geq 1000 \mathrm{~W} / \mathrm{m}^{2}$.
\end{abstract}

Keywords: solar cell efficiency, back contact, space charge region recombination.

Manuscript received 11.11.10; accepted for publication 02.12.10; published online 30.12.10.

\section{Introduction}

Commercial samples of up-to-date silicon back side contact (BSC) solar cells (SC) with $n$-type base, as well as modules produced on their basis, are characterized by the highest efficiency of photoelectrical energy conversion $\eta$ achieved till now that amounts to $22.4 \%$ for cells and $20.1 \%$ for modules [1]. As a rule, the thickness of a quasi-neutral base in such BSC SCs is much lower than the diffusion length of minority carriers, whereas the effective surface recombination velocity $S_{\text {eff }}$ on the front (nonmetallized illuminated) surface referred to the inner boundary of the near-surface space charge region (SCR) is minimized to the level that practically does not influence the value of $\eta$.

A detailed theoretical analysis of the factors that determine the effective surface recombination velocity $S_{\text {eff }}$ in silicon-based solar cells, where non-equilibrium electron-hole pairs recombine mainly via surface recombination centers, is performed in [2]. Using the self-consistent approach, it was demonstrated that, depending on the charge state of the near-surface SCR and the concentration of doping agents in the emitter and base region, the effective surface recombination velocity $S_{\text {eff }}$ can either grow or fall with increase in the concentration of non-equilibrium charge carriers in a quasi-neutral volume. Moreover, at sufficiently high injection levels, the velocity $S_{\text {eff }}$ saturates and is determined solely by parameters of surface recombination centers.

As known, the most effective way to eliminate surface recombination losses in BSC SCs is generation of isotype $n^{+}-n$ or $p^{+}-p$ junctions on their front surface, which limits the supply of non-equilibrium minority carriers to surface recombination centers [3, 4]. In presence of these junctions, the effective surface recombination velocity $S_{\text {eff }}$ is minimized due to a decrease of the true recombination velocity of nonequilibrium charge carriers via surface recombination centers [2], though, at high doping levels of the surface layer, the velocity $S_{\text {eff }}$ can rise due to an increase of the velocity of Auger recombination in this layer [3].

A number of works $[5,6]$ used another way of minimization of the negative influence of surface recombination losses on the efficiency of BSC SCs $\eta$, namely: formation of floating $p^{+}-n$ or $n^{+}-p$ junctions on their front surface, which limits the supply of non-equilibrium majority carriers to surface recombination centers. However, the experimental researches performed in [6] demonstrated that, though formation of a floating $n^{+}-p$ junction really resulted in an increase of the efficiency of BSC SCs $\eta$ under the 
standard spectral conditions AM1.5 at the irradiance $P_{L}=1000 \mathrm{~W} / \mathrm{m}^{2}$, a considerable (tens-fold) rise of the effective surface recombination velocity $S_{\text {eff }}$ was simultaneously observed. Such an anomalous behavior of the dependence $\eta\left(S_{\text {eff }}\right)$ could not be logically explained in the framework of the physical model of BSC SCs proposed in [6].

In the given work, it is shown that, in the presence of a floating $p^{+}-n$ or $n^{+}-p$ junction on the front (illuminated) surface, recombination in the SCR of the floating junction becomes a dominant recombination mechanism in BSC SCs at low light intensities. It is known that, in silicon solar cells of the common type, this recombination mechanism practically does not influence the magnitude of the short-circuit current, because photogeneration and collection of nonequilibrium electron-hole pairs take place mainly within the SCR of the collector junction, where non-equilibrium electrons and holes are separated by a strong electric field without recombination. But, in BSC SCs where the quasi-neutral base separates the regions of photogeneration and collection of non-equilibrium electron-holes pairs, recombination in the SCR of a floating $p^{+}-n$ or $n^{+}-p$ junction must essentially influence both the short-circuit current and the form of its spectral dependence measured at low light fluxes with the use of a monochromator.

Floating $p^{+}-n$ junction and isotype $n^{+}-n$ junction were compared as anti-recombinational barriers on the illuminated surface of $\mathrm{Si}$ solar cell with interdigitated back-side contacts in this work. Theoretical analysis and experimental research $[1,7]$ have been done for low illumination. Good agreement was achieved between theory and experiment for various illumination conditions.

\section{Theory}

The effective recombination velocity and its dependence on the illuminated surface dimensionless potential $y_{s}$, the bulk recombination time $\tau$ and the excess concentration on the boundary between SCR and the quasi-neutral region $\Delta p$ are calculated using model utilizing a deep recombination center near the middle of the band gap. In these conditions, the influence of this center is maximized. Assuming that recombination in SCR is determined by the same recombination center, we can write for $n$-type semiconductor:

$V_{S C}(\Delta p)=\frac{L_{D}}{\tau} \times$
$\times \int_{y_{s}}^{0} \frac{n_{0}}{\left[n_{0} e^{y}+\frac{C_{p}}{C_{n}}\left(p_{0}+\Delta p\right) e^{-y}\right] \sqrt{-y+\left(e^{y}-1\right)+\frac{\left(p_{0}+\Delta p\right)}{n_{0}} e^{-y}}} d y$, where $V_{S C}$ is the effective recombination velocity for SCR, $L_{D}=\left(\varepsilon_{0} \varepsilon_{s} k T / 2 q^{2} n_{0}\right)^{0.5}$ is the Debye length, $\tau$ is the bulk recombination time, $n_{0}$ and $p_{0}$ are equilibrium concentrations of electrons and holes, $C_{n}$ and $C_{p}$ are capture coefficients of the bulk recombination centers for electrons and holes.

Fig. 1 illustrates theoretical dependences of the effective recombination velocity in SCR $V_{S C}$ on the illuminated surface dimensionless potential $y_{s}$ for $n_{0}=10^{15} \mathrm{~cm}^{-3}, \quad p_{0}=10^{15} \mathrm{~cm}^{-3}, \quad \tau=10^{-5} \mathrm{~s} \quad$ and $C_{n}=C_{p}$. The excess concentration at the boundary between SCR and the quasi-neutral region $\Delta p$ differs. $V_{S C}$ rises with the rise of the band bending $y_{s}$ and saturates when $-y_{s}>(1 / 2) \ln \left(n_{0} /\left(p_{0}+\Delta p\right)\right)$. With the rise of $\Delta p$, saturated $V_{S C}$ decreases and the region of saturation expands. It is seen that when $\Delta p=10^{15} \mathrm{~cm}^{-3}$ (according to AM0 or AM1.5 conditions) $V_{S C}$ is five orders less than the value for absent photogeneration. It is important for $\mathrm{BSC} \mathrm{SC}$ to decrease the total recombination velocity for recombination through surface centers and recombination in SCR to the value $\leq 1 \mathrm{~cm} / \mathrm{s}$. In this case, surface recombination losses are less than the bulk recombination ones and the influence of surface recombination on the short-circuit current is negligible.

The analysis of $V_{S C}$ for the case of isotype $n-n^{+}$ junction near the frontal surface and values of $10^{15} \mathrm{~cm}^{-3}$ and $10^{-5} \mathrm{~s}$ for key parameters $n_{0}$ and $\tau$ shows the maximum value for $V_{S C}$ of the order of $1 \mathrm{~cm} / \mathrm{s}$ decreasing with the rise of $\tau$ and $\Delta p$. Thus, the recombination in SCR has no effect on photoelectrical parameters of BSC SC in the case of isotype $n-n^{+}$ junction.

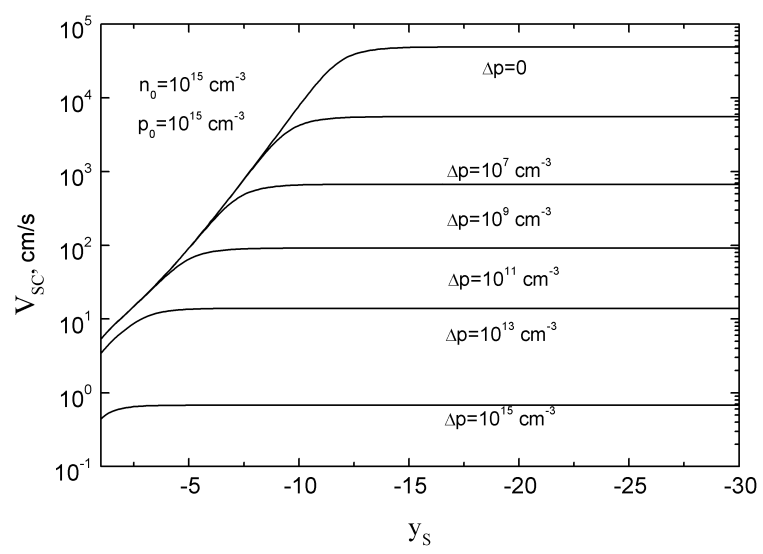

Fig. 1. The theoretical dependences of the effective recombination velocity in SCR $V_{S C}$ on the illuminated surface dimensionless potential $y_{s}$. 
The internal quantum efficiency $Q$ for Si BSC SC for the case of monochromatic illumination can be found by solving the diffusion equation with the following boundary conditions:

$j(x=0)=-\left(S+V_{S C}(\Delta p)\right) \Delta p(x=0)$,

$\Delta p(x=d)=0$,

where $S$ is the effective recombination velocity on the frontal surface of BSC SC.

The dependence of $\Delta p$ on the light level coefficient $M$ for the short-circuit regime is found from the equation of generation and recombination currents balance

$\left(S+V_{S C}(\Delta p)+\frac{d}{2 \tau}\right) \Delta p \approx\left[M J_{A M 0}-J_{S C}(M)\right] / q$.

In the case of AM0 conditions

$$
\begin{aligned}
& J_{A M O}=2 \pi q c\left(1-r_{s}\right)\left(R_{s} / R_{0}\right)^{2} \int_{0}^{\lambda_{x}} \frac{d \lambda}{\lambda^{4}\left[\exp \left(\frac{h c}{k T_{s} \lambda}\right)-1\right]}, \\
& J_{S C}(M)=2 \pi q c M\left(1-r_{s}\right)\left(R_{s} / R_{0}\right)^{2} \int_{0}^{\lambda_{x}} \frac{Q(\lambda) d \lambda}{\lambda^{4}\left[\exp \left(\frac{h c}{k T_{s} \lambda}\right)-1\right]},
\end{aligned}
$$

where $c$ is the velocity of light, $r_{s}$ is the reflection coefficient for the frontal surface of SC, $R_{S}$ is the radius of Sun, $R_{0}$ is the distance between Sun and Earth, $\lambda$ is the wavelength of the incident light, $\lambda_{x}$ is the photoelectric threshold, $h$ is Planck's constant, $k$ is the Boltzmann constant, $T_{S}$ is the temperature of Sun surface, $J_{S C}$ is the SC short-circuit current density, $M$ is the illumination level coefficient. The solar spectrum was assumed to be the blackbody spectrum with $T_{s}=5800 \mathrm{~K}$.

\section{Experiment}

Experimental research of isotype and floating junctions anti-recombinational properties was performed using $n$ type base BSC SCs. These SCs were fabricated on the basis of float zone $n$-type $\mathrm{Si}$ wafers; the resistivity was about $2 \mathrm{Ohm} \cdot \mathrm{cm}$. Process flow was the same excluding high-temperature processing. Phosphorus-doped isotype $n^{+}-n$ and boron-doped floating $p^{+}-n$ junctions on the $2 \mathrm{~cm}^{2}$ frontal illuminated surface were created in the course of high-temperature processing. 110-nm $\mathrm{SiO}_{2}$ film was created by $900{ }^{\circ} \mathrm{C}$ thermal oxidation to decrease optical losses. Aluminum ohmic contacts on the back surface of SC were created by vacuum deposition of $\mathrm{Al}$ on the surface of $n^{+}$- and $p^{+}$-doped regions through windows in $\mathrm{SiO}_{2}$-film. These windows were created by the photolithographic method. After the photolithography SCs were heated in vacuum at $430{ }^{\circ} \mathrm{C}$ to decrease the resistance of contacts.

Three sets of BSC SC were fabricated. The differences in temperature and duration of the high- temperature processing produced minority carrier diffusion lengths $L$ of 250, 500 and $1100 \mu \mathrm{m}$ in sets.

Short-circuit current spectra were measured in the automatic mode with constant illumination. Phototechnical parameters (short-circuit current, opencircuit voltage) were measured under outdoor conditions (AM1.5). The irradiance of SC surface was widely modified using neutral filters.

\section{Results and discussion}

Fig. 2 shows theoretical and experimental dependences of the internal quantum efficiency $Q$ for the floating $p-n$ junction and for the isotype $n-n^{+}$junction near the illuminated surface. There are lines for theoretical dependences and points for the experimental ones. Experimental values of $Q$ were measured using a monochromator at low light intensities. Fig. 2 indicates that $Q(\lambda)$ strongly decreases with $\lambda$ for the floating $p-n$ junction, this dependence corresponds to large $V_{S C}$. Dependences for additional constant illumination confirm this correspondence. These dependences indicate the rise of $Q(\lambda)$ in short-wave region determined by $V_{S C}$ decrease. In the case of the isotype $n-n^{+}$ junction, there is no short-wave decrease. Fig. 3 shows theoretical and experimental dependences of shortcircuit current $I_{S C}$ on illumination level $M$ for the $n-n^{+}$ junction and for the $p-n$ junction near the illuminated surface. The theoretical dependences are built using (4) and (5) with parameters for AM1.5. It is seen that for low excitation floating $p-n$ junction short-circuit current demonstrates ultra-linear rise, and this current is less than isotype $n-n^{+}$junction short-circuit current. It means that $V_{S C}$ decreases with rise of $M$. The figure shows good agreement between theory and experiment.

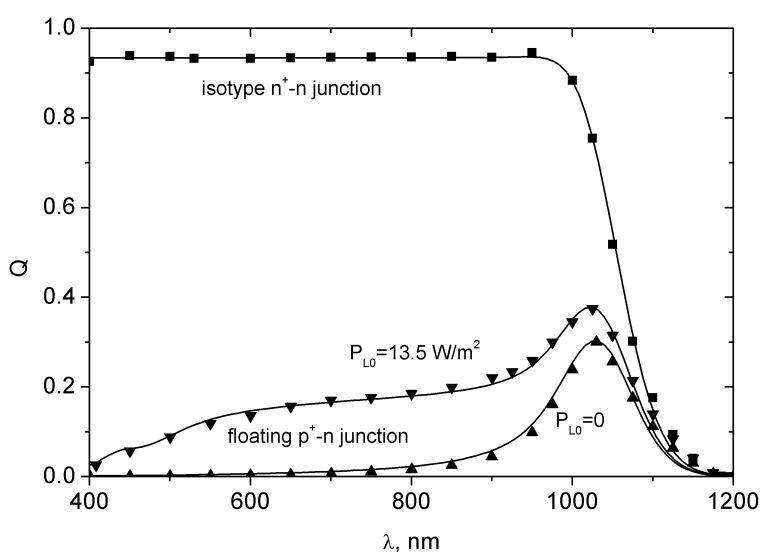

Fig. 2. Theoretical (lines) and experimental (points) wavelength dependences of the internal quantum efficiency $Q$ for the floating $p-n$ junction and for the isotype $n-n^{+}$ junction near the illuminated surface. 


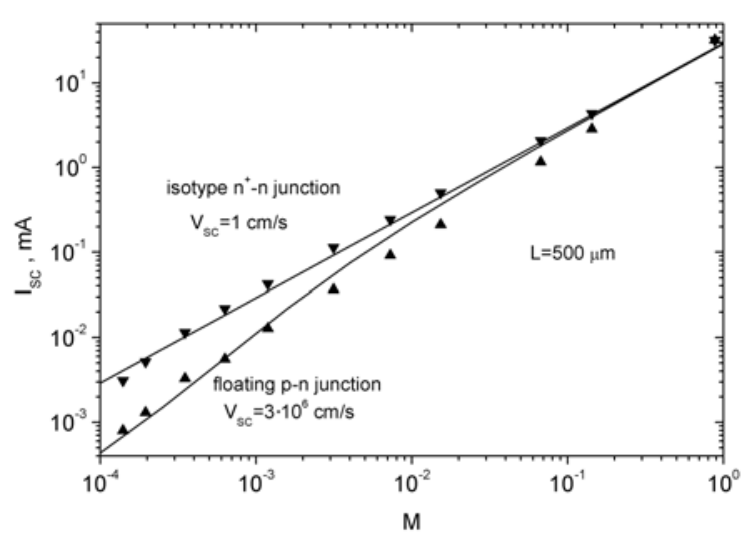

Fig. 3. Theoretical (lines) and experimental (points) dependences of short-circuit current $J_{S C}$ on the illumination level $M$.

It is necessary to mention that SCR recombination with the current-voltage ideality factor $\beta=2$ has significant influence on the open-circuit voltage $V_{O C}$ in both conventional SCs and BSC SCs. In the case of floating $p-n$ junction near the illuminated surface, $V_{O C}$ is influenced by the BSC recombination near both frontal and rear surfaces. In the case of isotype $n-n^{+}$ junction near the illuminated surface, there is significant SCR recombination near the rear surface and significantly lower recombination near the illuminated surface. However, experimental $V_{O C}(M)$ dependences do not agree with the theoretical ones taking into consideration only BSC recombination with the currentvoltage ideality factor $\beta=2$. Tunnel recombination has to be taken into consideration. We assume that tunnel recombination takes place between $p^{+}$and $n^{+}$regions on the rear surface (for samples with colliding $p^{+}$and $n^{+}$ regions). We use the following equation to reach the agreement between the experiment and the theory for open-circuit voltage:

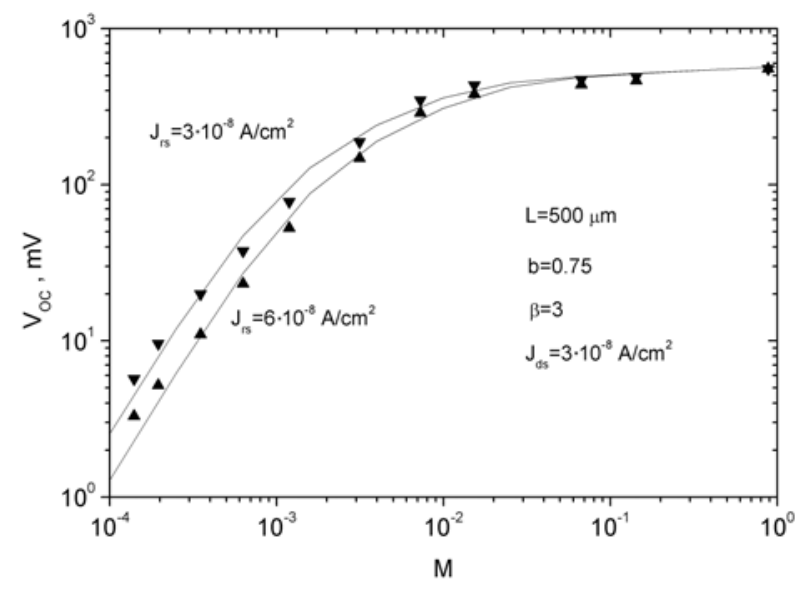

Fig. 4. Theoretical (lines) and experimental (points) dependences $V_{O C}(M)$.

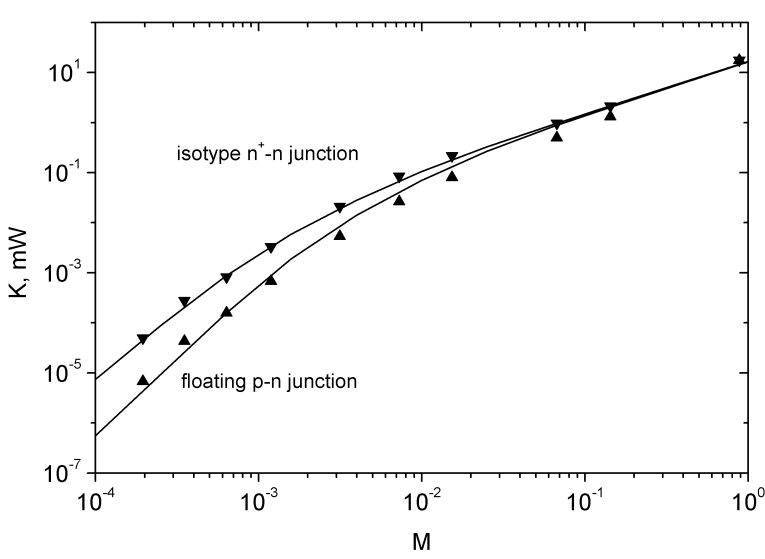

Fig. 5. Theoretical (lines) and experimental (points) dependences for $K=J_{S C} V_{O C}=P / F F$ on the illumination level $M$.

$$
J_{d s}\left(\exp \left(\frac{q V_{O C}}{k T}\right)-1\right)+\frac{J_{r s}}{M^{b}}\left(\exp \left(\frac{q V_{O C}}{\beta k T}\right)-1\right)=q J_{S C}(M) .
$$

Here $q$ is elementary electronic charge, $k$ is Boltzmann constant, $T$ is the temperature, $J_{d s}$ and $J_{r s}$ are densities of diffusion and recombination saturation currents, $b$ is a coefficient less than 1 and $\beta>2$.

Fig. 4 shows theoretical and experimental dependences $V_{O C}(M)$. One can see that, in low illumination region, $V_{O C}$ for the floating $p-n$ junction is less than $V_{O C}$ for the isotype $n^{+}-n$ junction near the illuminated surface. There is a good agreement between theory and experiment.

Fig. 5 demonstrates theoretical and experimental dependences for $K(M)=J_{S C} V_{O C}=P / F F$. Here $P$ is the photoconversion power and $F F$ is the fill factor of current-voltage characteristics. The figure shows that the photoconversion efficiency for the floating $p-n$

junction is significantly less than for the isotype $n^{+}-n$ junction near the illuminated surface in the low illumination region.

Thus, it can be concluded that the use of floating $p-n$ junctions as anti-recombinational barriers on the frontal (illuminated) surface of $\mathrm{Si}$ solar cells with back side contacts can be effective to decrease surface recombination losses only in the case of high surface irradiance $P_{L}\left(P_{L} \geq 1000 \mathrm{~W} / \mathrm{m}^{2}\right)$. In the case of lower illumination, the use of these junctions is not reasonable and can significantly reduce the photoconversion efficiency.

\section{Conclusions}

Theoretical and experimental studies demonstrate that the use of floating $p-n$ junctions as antirecombinational barriers on the frontal (illuminated) 
surface of Si solar cells with back side contacts can be effective only when the non-equilibrium electron-hole pairs injection is high and the band bending in the floating junction is significantly moved under illumination. In the case of low illumination, the recombination velocity in SCR of the floating junction sharply rises. This rise significantly decreases the shortcircuit current and open-circuit voltage as compared with the short-circuit current and the open-circuit voltage of SCs with the isotype $n^{+}-n$ junctions on the illuminated surface used for minimization of surface recombination losses. Thus, the use of floating $p-n$ junctions as anti-recombinational barriers in weakly illuminated $\mathrm{Si}$ photovoltaic devices (solar cells, photodetectors, photosensors) is not reasonable. However, there are no such disadvantages in the case of isotype $n^{+}-n$ junctions.

\section{References}

1. D. De Ceuster, P. Cousins, D. Rose, D. Visente, P. Tipones, W. Mulligan // Proc. 23th European
Photovoltaic Solar Energy Conference, 2007, Sept. 3-7, Milan, p. 816-819 (2007).

2. A.P. Gorban, V.P. Kostylyov, A.V. Sachenko, A.A. Serba, I.O. Sokolovskyi // Ukrainsky fizychny zhurnal, 51(6), p. 599-606 (2006), in Ukrainian.

3. M.I. Yernaux, C. Battochio, P. Verlinden, F. Van De Wiele // Solar Sells, 13, p. 83-97 (1984).

4. W.P. Mulligan, D.H. Rose, M.J. Cudzinovic, D.M. De Ceuster, K.R. McIntosh, D.D. Smith, R.M. Swanson // Proc. 19th European Photovoltaic Solar Energy Conference, 2004, June 7-11, Paris, p. 387-390 (2004).

5. J. Dicker, J.O. Schumacher, S.W. Glunz, W. Warta // Proc. 2nd World Conference on Photovoltaic Solar Energy Conversion, 1998, July 6-10, Vienna, p. 95-99 (1998).

6. T. Nagashima, K. Hokoi, K. Okumura, M. Yamaguchi // Proc. 20th European Photovoltaic Solar Energy Conference, 2005, June 6-10, Barselona, p. 163-166 (2005).

7. R.M. Swanson, Point-contact solar cells: modeling and experiment // Solar Cells, 17(1), p. 85-118 (1986). 